\title{
Análisis comparativo de las propiedades fisicoquímicas y capacidad antioxidante de un morfotipo de orégano (Origanum vulgare L.) cultivado en dos localidades de la sierra ecuatoriana
}

\section{Comparative analysis of the physicochemical properties and antioxidant capacity of a morphotype of oregano (Origanum vulgare L.) cultivated in two locations of the ecuadorian sierra}

Tamara Fukalova F. ${ }^{*}$, Beatriz Brito G. ${ }^{2}$, Tania Novoa C. ${ }^{3}$, Evelyn Sagñay T. ${ }^{4}$, Iván Samaniego M. ${ }^{5}$, Marcelo Tacán P. ${ }^{6}$, Iván Tapia C. ${ }^{7}$

${ }^{1}$ Universidad Central del Ecuador, Facultad de Ciencias Químicas. Francisco Viteri y Gilberto Gatto Sobral, Quito, Ecuador. $\mathrm{CP}: 170521$.

凶tfukalova@uce.edu.ec

https://orcid.org/0000-0001-9547-0002

${ }^{2}$ Instituto Nacional de Investigaciones Agropecuarias, Estación Experimental Santa Catalina. Panamericana Sur km. 1 vía Tambillo, cantón Mejía, Pichincha, Ecuador. CP: 171108.

$凶$ beatriz.brito@iniap.gob.ec

https://orcid.org/0000-0002-3581-6777

${ }^{3}$ Universidad Central del Ecuador, Facultad de Ciencias Químicas. Francisco Viteri y Gilberto Gatto Sobral, Quito, Ecuador. $\mathrm{CP}: 170521$.

凶tpnovoa@uce.edu.ec https://orcid.org/0000-0002-2103-829X

${ }^{4}$ Universidad Central del Ecuador, Facultad de Ciencias Químicas. Francisco Viteri y Gilberto Gatto Sobral, Quito, Ecuador. $\mathrm{CP}: 170521$.

凶epsagnay@uce.edu.ec

https://orcid.org/0000-0002-3325-6198

${ }^{5}$ Instituto Nacional de Investigaciones Agropecuarias, Estación Experimental Santa Catalina. Panamericana Sur km. 1 vía Tambillo, cantón Mejía, Pichincha, Ecuador. CP: 171108.

\ivan.samaniego@iniap.gob.ec

https://orcid.org/0000-0001-9274-7037

${ }^{6}$ Instituto Nacional de Investigaciones Agropecuarias, Estación Experimental Santa Catalina. Panamericana Sur km. 1 vía Tambillo, cantón Mejía, Pichincha, Ecuador. CP: 171108.

凶marcelo.tacan@iniap.gob.ec

https://orcid.org/0000-0002-6775-1445

${ }^{7}$ Universidad Central del Ecuador, Facultad de Ciencias Químicas. Francisco Viteri y Gilberto Gatto Sobral, Quito, Ecuador. $\mathrm{CP}: 170521$.

凶iltapia@uce.edu.ec

https://orcid.org/0000-0001-9305-4793 


\section{Resumen}

El orégano (Origanum vulgare L.) es una especie introducida en la flora ecuatoriana. Por sus propiedades es muy apreciada y consumida, por lo que es importante determinar la naturaleza de sus beneficios. En este estudio se evaluó la composición fisicoquímica y el contenido de los compuestos funcionales responsables de la actividad antioxidante de la planta en la accesión ECU-20229 del Banco de Germoplasma del Instituto Nacional de Investigaciones Agropecuarias (INIAP). El cultivo se realizó en Italquí provincia de Imbabura y en Tumbaco provincia de Pichincha. Se analizó la planta entera y sus partes (raíz, tallo y hojas), con la finalidad de determinar dónde se encuentra el mayor contenido de proteína, grasa, minerales y los compuestos funcionales. Se realizó el análisis proximal, concentración de minerales, polifenoles y flavonoides totales, así como la capacidad antioxidante. El contenido de los compuestos analizados fue estadísticamente diferente entre las partes de la planta analizadas, y entre los sitios de su cosecha. La concentración de proteína, grasa y carbohidratos fue superior en las hojas que en las otras partes de la planta. La concentración de estos compuestos, así como de los macro y microminerales, fue mayor en Italquí, al igual que el contenido de polifenoles y flavonoides totales. La capacidad de antioxidante fue mayor en Tumbaco. En este estudio se han confirmado las propiedades nutritivas descritas para esta planta en beneficio de la salud humana y animal, pero además se aporta información sobre el valor agregado a las partes de la planta que regularmente son desaprovechadas.

Palabras clave: ambiente, calidad química, compuestos funcionales.

\section{Abstract}

Oregano (Origanum. vulgare L.) is a species introduced into the Ecuadorian flora. This species due to its culinary properties is highly appreciated and consumed, and therefore the nature of its benefits is important to be determined. In this study, the chemical composition and the functional compounds content responsible for the antioxidant activity of the plant were evaluated in the oregano accession ECU-20229 from the Germplasm Bank of the National Institute for Agriculture Research (INIAP). The oregano was grown in Italquí-province of Imbabura and Tumbaco-province of Pichincha. The whole plant and the plant parts root, stem and leaves were analyzed to determine the content of protein, fatty compounds, minerals and functional compounds. The proximal analysis, the concentration of minerals, polyphenols and flavonoids, as well as the antioxidant capacity were performed. Content of compounds were statistically different among plant parts as well as among sites. Concentration of protein, fatty compounds and carbohydrates were higher on leaves than the other parts of the plant. Concentration of these compounds as well as the micro and macro-minerals were higher in Italquí than in Tumbaco, as well as the content of the total polyphenols and flavonoids. The antioxidant capacity was higher in Tumbaco. The nutritional properties that have been described for this plant for the benefit of human and animal health have been confirmed, and in this research an adding value has been incorporated to the plant parts that are regularly not used.

Keywords: environment, chemical quality, functional compounds.

\section{Introducción}

En la actualidad, la humanidad busca maneras de incorporar prácticas saludables a su vida diaria. En consecuencia, las plantas aromáticas y medicinales, $y$ las de origen vegetal han originado un interés creciente de consumo, debido a los diversos beneficios para la salud humana y animal. La planta de orégano (Origanum vulgare L.) al ser empleada en recetas culinarias forma parte de la alimentación humana. Los alimentos de origen vegetal se caracterizan por la presencia de meta- 
bolitos secundarios, entre los cuales se destacan los compuestos bioactivos y funcionales. El orégano es una especie que se usa como condimento gastronómico y es apreciado por su función medicinal, adjudicándole beneficios como tónico, calmante, estimulante del apetito, entre otros (de la Torre, 2008). Varias investigaciones han demostrado que tiene efectos benéficos en la salud de las personas (Koksal et al., 2010). El orégano es una planta aromática originaria de Europa, cultivada en varias regiones del mundo, por lo que existen diferentes variedades con característica propias. En Ecuador tenemos una especie introducida y por sus características organolépticas es muy apreciada y consumida. Sin embargo, de todas sus partes estructurales (raíz, hojas, tallo), las más utilizadas son las hojas, tanto frescas como secas. Según el Instituto Nacional de Investigaciones Agropecuarias (Allauca et al., 2013), ciertas plantas medicinales y aromáticas, el orégano entre ellas, se encuentran cultivadas en huertos caseros que suplen las necesidades alimenticias y medicinales de las familias que la cultivan, usando el conocimiento ancestral; es por ello que en el país no se han realizado muchas investigaciones sobre los beneficios de la planta de orégano producida a nivel nacional, en cuanto a su composición nutricional, la presencia de minerales y su capacidad antioxidante.

Impulsados por el redescubrimiento de plantas provechosas presentes en la flora ecuatoriana, este trabajo buscó evaluar la composición química nutricional y el contenido de compuestos bioactivos y funcionales responsables de la actividad antioxidante de la planta orégano cultivada en dos zonas medioambientales de las provincias de Imbabura y Pichincha, así como aportar un valor agregado a las partes desaprovechadas que son el tallo y la raíz.

\section{Materiales y métodos}

Se trabajó con las plantas de orégano $(O$. vulgare L.) ECU-20229, conservadas en el Banco de Germoplasma del Instituto Nacional de Investigaciones Agropecuarias que fueron multiplicadas en dos localidades. En la provincia de Pichincha, cantón Quito, parroquia Tumbaco, localidad Granja Experimental Tumbaco del INIAP, con una temperatura promedio de $16,2{ }^{\circ} \mathrm{C}$; altitud 2.348 m s.n.m.; coordenadas de la recolección: latitud $00^{\circ} 13^{\prime} 00^{\prime} \mathrm{S}$, longitud $78^{\circ} 24^{\prime} 01^{\prime \prime} \mathrm{O}$. En la provincia de Imbabura, cantón Cotacachi, parroquia El Sagrario, localidad Italquí, con una temperatura promedio de $15{ }^{\circ} \mathrm{C}$; altitud 2.684 m s.n.m.; coordenadas de la recolección: latitud $00^{\circ} 18^{\prime} 06^{\prime}$ 'S, longitud 78 $18^{\circ} 53^{\prime \prime} \mathrm{O}$.

Con las plantas en estado de madurez fisiológico y de tamaño mediano, se realizó el muestreo al azar. Una vez realizada la limpieza para eliminar los residuos de tierra, las plantas cosechadas se distribuyeron de la siguiente manera: para el análisis proximal y fisicoquímico (determinación de macrocomponentes) $300 \mathrm{~g}$ de planta entera fresca y otros $300 \mathrm{~g}$ de los cuales fueron separados la raíz, tallo y hojas. Para realizar estos análisis la planta se sometió a un proceso de secado en una estufa a $65^{\circ} \mathrm{C}$ (LAB LINE Imperial V) por 12 horas, las muestras secas fueron molidas y almacenadas en frascos de plástico hasta el momento de los análisis. Para el análisis de los compuestos funcionales y la capacidad antioxidante fueron congeladas a $-18^{\circ} \mathrm{C}$ (Elecrolux. EC306NBHW) y luego se liofilizaron durante 72 horas a $-30{ }^{\circ} \mathrm{C}$ y $0,1 \mathrm{~Pa}$ (Lab Kits FD-18-MR), las muestras liofilizadas se molieron (RETSCH ZM-200) y almacenaron en frascos plásticos debidamente rotulados.

\subsection{Análisis fisicoquímicos}

El análisis proximal que comprende la determinación de los porcentajes se realizó en la planta entera y en sus partes. Los métodos de análisis aplicados se encuentran descritos en el manual del laboratorio (INIAP, 2017). Se consideraron los siguientes parámetros: la humedad obtenida por evaporización del agua al someter la muestra al calor en una estufa de aire forzado (Lab-Line IMPERIAL V) (MO-LSAIA-01.01); la proteína se analizó por el método Kjeldahl (FOSS Tecator) (MO-LSAIA-01.04); la fibra obtenida en el analizador (LABCONCO) es el residuo de una digestión ácida y alcalina (MO-LSAIA-01.05); el contenido graso se extrajo con hexano en un analizador (FOSS Soxtec ${ }^{\mathrm{TM}}$ 2043) (MOLSAIA-01.03); las cenizas se obtuvieron por la incineración en una mufla (Thermolyne 48000 Furnace) (MO-LSAIA-01.02); los carbohidratos totales se obtuvieron por diferencia del $100 \%$ 
del contenido de fibra, grasa, proteína y cenizas (MO-LSAIA-01.06). En el contenido de macro y microminerales, se determinó el fósforo por espectrofotometría UV/VIS (Shimadzu Uv-2600) (MO-LSAIA-03.01) y por espectrofotometría de absorción atómica (Shimadzu AA-7000) el Ca, $\mathrm{Mg}, \mathrm{Na}, \mathrm{K}, \mathrm{Cu}, \mathrm{Fe}, \mathrm{Mn}, \mathrm{Zn}$ (MO-LSAIA 03.02).

\subsection{Análisis de compuestos funcionales}

Este análisis se efectuó con las muestras liofilizadas, se realizó la extracción en una mezcla metanol/agua/ácido fórmico en un baño de ultrasonido (Cole Parmer 8892-MHT), se centrifugó (Damon/IEC) y una vez separado el sobrenadante la extracción se repitió por cinco veces. En el sobrenadante se analizaron los compuestos funcionales por espectrofotometría UV/VIS (Shimadzu Uv-2600). Para los polifenoles totales, se midió la absorbancia de los extractos a $760 \mathrm{~nm}$ según el método de Cross et al. (1982), utilizando una curva de calibración de ácido gálico (TraceCERT, Supelco). Los flavonoides totales se determinaron por el método de Zhishen et al. (1999), se midió la absorbancia a $490 \mathrm{~nm}$, con una curva de calibración de catequina (Sigma-Aldrich).

\subsection{Análisis de la capacidad antioxidante}

La capacidad antioxidante en las muestras liofilizadas se determinó mediante dos métodos: catión radical del ácido 2,2-azinobis-(3-etilenbenzotiazolino-6-sulfonico) $\left(\mathrm{ABTS}^{+}\right)$sugerido por Re et al. (1998) y la reducción de los iones ferroso $\mathrm{Fe}^{2+}$ a iones férrico $\mathrm{Fe}^{3+} \mathrm{FRAP}$ ), utilizando la metodología propuesta por (Yen \& Chen,
1995). Para ambos métodos se utilizó una curva de calibración de trolox (Sigma-Aldrich) como estándar en un rango de concentraciones de $0 \mathrm{a}$ $600 \mu \mathrm{g} / \mathrm{ml}$. El cambio de color se midió en espectrofotómetro UV/VIS (Shimadzu Uv-2600).

Para la cuantificación de los compuestos funcionales y la capacidad antioxidante se realizó previamente la determinación de la linealidad, exactitud y precisión de los métodos, con los parámetros del límite de detección, porcentaje de recuperación y la repetibilidad.

\subsection{Análisis estadístico}

Para cada respuesta experimental se realizó un análisis estadístico de los resultados obtenidos para cada tratamiento: Factor A (planta entera, raíz, tallo y hojas) y el Factor B (dos localidades), con un análisis de varianza ANOVA al $95 \%$ de confianza, en los tratamientos significativos se realizó una separación de medias con la prueba de Tukey al 95\% (programa IBM SPSS Statistic, versión 20).

\section{Resultados}

\subsection{Análisis proximal}

Los resultados en la planta entera y sus partes se presentan en la Tabla 1. En la planta entera los valores de todos los parámetros fueron similares para las dos localidades, a excepción del contenido de proteína que resultó ser mayor en el orégano de Pichincha

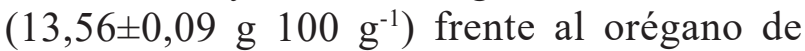

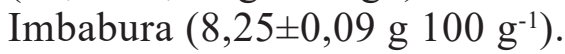

Tabla 1. Análisis proximal del orégano ECU-20229 de las localidades de Pichincha e Imbabura, resultados en base seca

\begin{tabular}{|c|c|c|c|c|c|c|c|}
\hline Localidad & Trat. & $\begin{array}{l}\text { Humedad } \\
\left(\mathrm{g} 100 \mathrm{~g}^{-1}\right)\end{array}$ & $\begin{array}{l}\text { Proteína } \\
\left({\left.\mathrm{g} 100 \mathrm{~g}^{-1}\right)}\right.\end{array}$ & $\begin{array}{c}\text { Fibra } \\
\left(\mathrm{g} 100 \mathrm{~g}^{-1}\right)\end{array}$ & $\begin{array}{l}\text { Ext. Etéreo } \\
\left(\mathrm{g} 100 \mathrm{~g}^{-1}\right)\end{array}$ & $\begin{array}{l}\text { Cenizas } \\
\left({\left.\mathrm{g} 100 \mathrm{~g}^{-1}\right)}\right.\end{array}$ & $\begin{array}{c}\text { Carbohidratos } \\
\left(\mathrm{g} 100 \mathrm{~g}^{-1}\right)\end{array}$ \\
\hline \multirow{4}{*}{$\begin{array}{l}\text { Pichincha- } \\
\text { Tumbaco }\end{array}$} & $\mathbf{P E}$ & $77,91 \pm 0,02$ & $13,56 \pm 0,09$ & $27,88 \pm 0,48$ & $1,55 \pm 0,02$ & $9,93 \pm 0,08$ & $47,09 \pm 0,33$ \\
\hline & $T_{1}$ & $69,83 \pm 0,03$ & $6,38 \pm 0,20$ & $42,55 \pm 1,13$ & $0,68 \pm 0,01$ & $11,02 \pm 0,39$ & $39,38 \pm 1,21$ \\
\hline & $\mathbf{T}_{2}$ & $74,10 \pm 0,04$ & $7,93 \pm 0,22$ & $45,95 \pm 1,31$ & $0,73 \pm 0,02$ & $10,63 \pm 0,47$ & $34,76 \pm 0,78$ \\
\hline & $\mathbf{T}_{3}$ & $69,05 \pm 0,02$ & $18,61 \pm 0,12$ & $11,31 \pm 0,16$ & $2,89 \pm 0,05$ & $12,92 \pm 0,02$ & $54,28 \pm 0,33$ \\
\hline \multirow{4}{*}{$\begin{array}{l}\text { Imbabura- } \\
\text { Italquí }\end{array}$} & $\mathbf{P E}$ & $75,89 \pm 0,40$ & $8,27 \pm 0,09$ & $28,62 \pm 0,03$ & $1,18 \pm 0,06$ & $10,77 \pm 0,08$ & $47,34 \pm 0,07$ \\
\hline & $T_{1}$ & $68,81 \pm 0,33$ & $6,41 \pm 0,29$ & $63,41 \pm 0,19$ & $0,86 \pm 0,06$ & $10,18 \pm 0,35$ & $18,53 \pm 0,22$ \\
\hline & $\mathbf{T}_{2}$ & $72,11 \pm 0,08$ & $9,03 \pm 0,05$ & $51,53 \pm 0,20$ & $0,80 \pm 0,01$ & $13,32 \pm 0,06$ & $24,40 \pm 0,08$ \\
\hline & $T_{3}$ & $74,10 \pm 0,14$ & $19,58 \pm 0,36$ & $10,21 \pm 0,16$ & $1,93 \pm 0,06$ & $12,06 \pm 0,17$ & $50,49 \pm 0,19$ \\
\hline
\end{tabular}

PE: planta entera; $\mathrm{T}_{1}$ : raíz; $\mathrm{T}_{2}$ : tallo; $\mathrm{T}_{3}$ : hojas $; \mathrm{n}=3$ 
El mayor contenido de proteína se obtuvo en las hojas con 18,61 \% y 19,58 \%, seguido del tallo con $13,56 \%$ y $8,27 \%$ en el orégano de Pichincha e Imbabura, respectivamente. El mayor contenido de fibra con $63,41 \%$ se encuentra en la raíz del orégano de Imbabura, seguido del tallo con 51,53 $\%$, el menor contenido se obtuvo en las hojas para el orégano de ambas localidades. Para las cenizas el mayor contenido se encontró en las hojas con $12,92 \%$ del orégano de Pichincha y en el tallo $13,32 \%$ del orégano de Imbabura. En los carbohidratos totales, el mayor contenido se encuentra en las hojas con 54,28 \% de Pichincha y 50,49\% en las cosechadas en Imbabura; por el contrario, en la raíz prevaleció en el orégano de Pichincha con $39,38 \%$ y fue menor en el orégano de Imbabura con $18,52 \%$. El mayor contenido del extracto eté- reo (grasa y pigmentos) fue mayor en las hojas con 2,89\% (Pichincha) y 1,93\% (Imbabura).

Siendo el valor $\mathrm{F}$ experimental mayor al valor $\mathrm{F}$ tabulado, el análisis de varianza indica, para todos los casos, diferencias estadísticas en composición nutricional (factor A) en función de los ambientes (factor B) para raíz, tallo y hojas.

\subsection{Análisis de minerales}

Macrominerales. En la Figura 1 se presentan los resultados expresados como la media y la desviación estándar $(\mathrm{n}=3)$ de los macrominerales (calcio, magnesio, sodio, potasio y fósforo) en los tratamientos que son el Factor A (partes de la planta) y en las dos localidades que corresponde al Factor B.

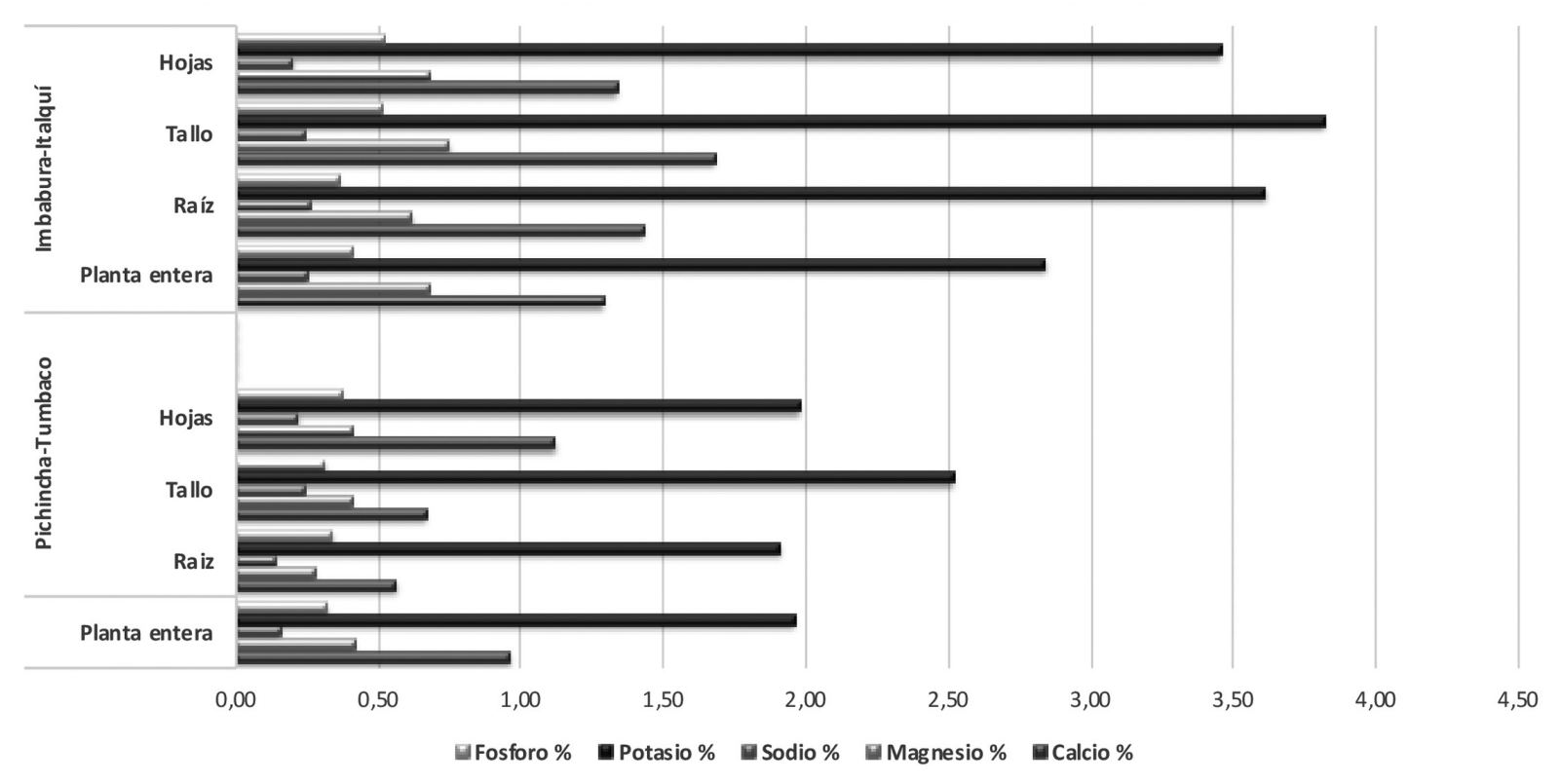

Figura 1. Porcentaje de macrominerales de planta de orégano ECU-20229 de Pichincha e Imbabura, resultados en base seca.

En la planta entera como en sus partes individuales (raíz, tallo, hoja) se registra mayor porcentaje, en orden descendente, de los macrominerales potasio, calcio, magnesio, fósforo y sodio. Este comportamiento es igual en ambas localidades, sin embargo, los porcentajes son mayores en las plantas establecidas en Italquí (Figura 1). El potasio $(2,84 \%)$ y el calcio $(1,29 \%)$ son los minerales de mayor presencia en la planta entera de Italquí frente a la planta entera de Tumbaco con 1,96\% y $0,96 \%$ para el potasio y el calcio, respectivamente.
Se determinó la parte de la planta que presenta el mayor contenido de estos elementos, analizándose por separado en cada uno de sus órganos, permitiendo identificar que el elemento con mayor cantidad es el potasio y abunda en el tallo con 2,52\% en Tumbaco y 3,82\% en Italquí. El elemento con menor presencia en las partes del orégano es el sodio, con cantidades similares en el tallo y en las hojas con 0,24 y 0,22 $\%$, respectivamente, en Tumbaco; 0,24 y $0,20 \%$ en Italquí, entra por igual en todas las partes de 
la planta. El magnesio y el fósforo tienen valores mayores en el orégano cultivado en Italquí de la provincia de Imbabura.

Microminerales. De los microminerales (cobre, manganeso y zinc) el manganeso predo- minó tanto en la planta entera como en las partes individuales, seguido por el zinc para el orégano de Tumbaco, como se muestra en la Figura 2. Mientras que para el orégano de Italquí, el manganeso predominó en la planta entera, la raíz y tallo y el zinc predominó en las hojas.

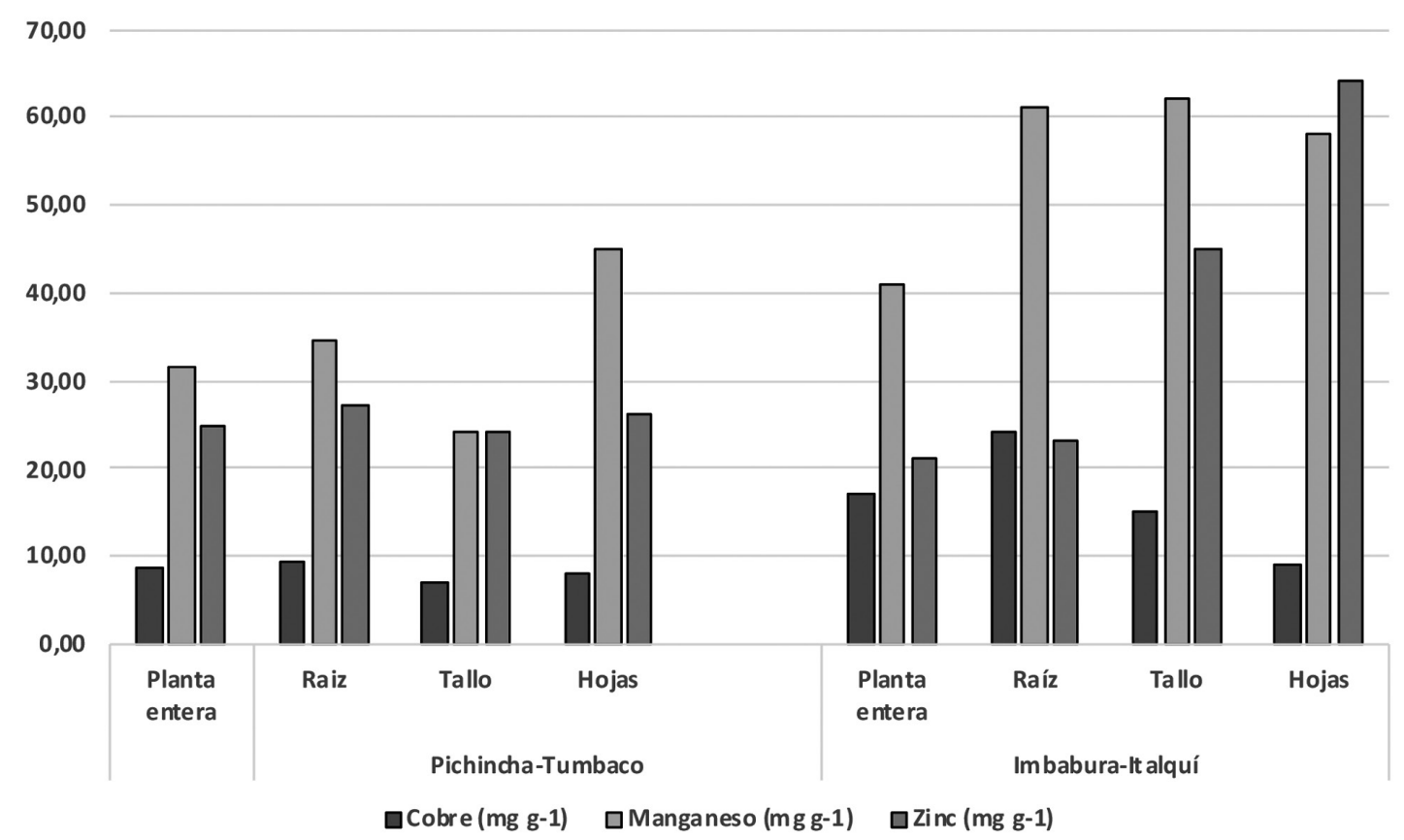

Figura 2. Concentraciones de microminerales de planta de orégano ECU-20229 de Pichincha e Imbabura, resultados en base seca.

En las partes individuales (raíz, tallo, hoja) frente a la planta entera se registran mayores cantidades de los microminerales como el zinc y el manganeso. Este comportamiento es igual en ambas lo- calidades, sin embargo, la mayor concentración de zinc corresponde a las hojas con $64,00 \mathrm{mg} \mathrm{g}^{-1}$ en las hojas y de manganeso con $62,00 \mathrm{mg} \mathrm{g}^{-1}$ en el tallo del orégano establecido en Italquí (Figura 2).

\section{Hierro ( $\propto$ g g-1)}

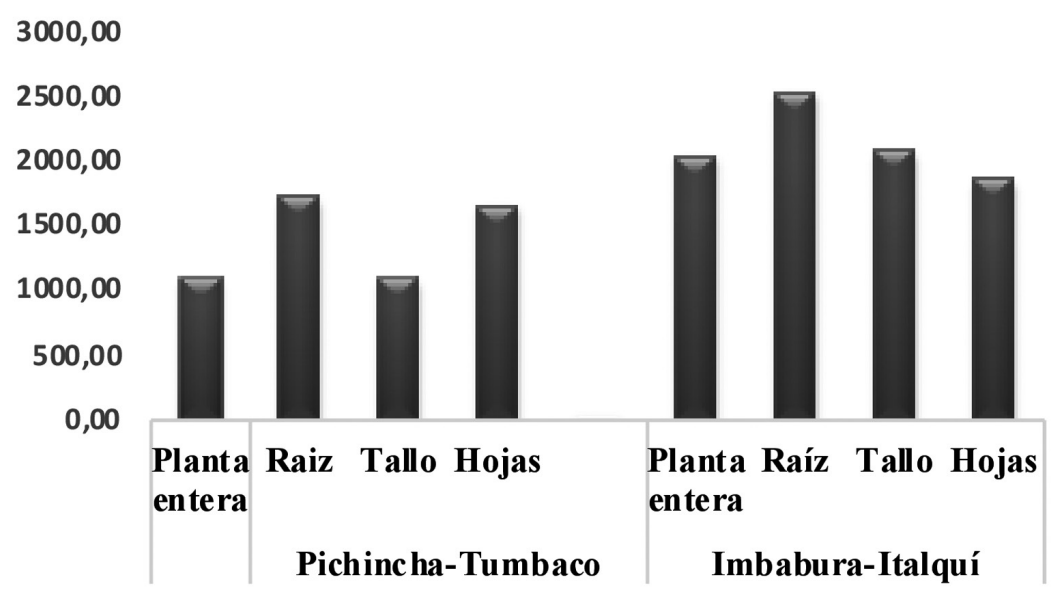

Figura 3. Concentración de hierro en planta de orégano ECU-20229 de Pichincha e Imbabura, resultados en base seca. 
En la Figura 3, se observa que se destaca el hierro por su mayor contenido, correspondiendo a la raíz del orégano de Italquí con $2.518 \mu \mathrm{g}$ $\mathrm{g}^{-1} \mathrm{y}$ de Tumbaco con $1.706 \mu \mathrm{g} \mathrm{g}^{-1}$. En las hojas presentaron valores de $1.637 \mu \mathrm{g} \mathrm{g}^{-1}$ en Tumbaco y $1.853 \mu \mathrm{g} \mathrm{g}^{-1}$ en Italquí.

\subsection{Análisis de compuestos funcionales y capa- cidad antioxidante}

Con el propósito de asegurar que los resultados sean reproducibles y confiables se realizó la adaptación del método de análisis de polifenoles totales, flavonoides totales y capacidad antioxidante $\left(\mathrm{ABTS}^{+}\right.$y FRAP), estudiándose la linealidad, precisión y exactitud. La curva de calibración presentó un alto coeficiente de correlación para la linealidad $\left(R^{2}>0,995\right)$. La exactitud con cinco ciclos de extracción evidenció una recuperación $98,47 \%$ de polifenoles totales y $95,54 \%$ de flavonoides totales. La precisión permitió asegurar la repetibilidad de los métodos, con el coeficiente de variación 1,09 \% (polifenoles totales) y $1,33 \%$ (flavonoides totales). Para fines comparativos los datos experimentales de flavonoides fueron transformados, de $\mathrm{mg}$ catequina $\cdot \mathrm{g}^{-1}$ a $\mathrm{mg}$ ácido gálico $\cdot \mathrm{g}^{-1}$ utilizando una relación de los pesos moleculares.

En la Tabla 2 se presentan los resultados de los compuestos funcionales y la capacidad antioxidante. Se puede observar que el orégano de Italquí tiene mayor contenido de polifenoles y flavonoides, tanto en la planta entera como en sus partes, con el mayor contenido en las hojas con 145,24 y 131,71 ( $\left.\mathrm{mg} \mathrm{g}^{-1}\right)$, los valores que se obtuvieron en Tumbaco fueron de 93,56 y 83,00 (mg g $\left.{ }^{-1}\right)$, respectivamente. Por el contrario, los contenidos menores son en el tallo y las raíces. El aporte en los valores de la planta entera se debe al alto contenido que tienen las hojas.

Tabla 2. Compuestos funcionales y capacidad antioxidante del orégano ECU-20229 de las localidades de Pichincha e Imbabura, resultados en base seca

\begin{tabular}{|c|c|c|c|c|c|}
\hline Localidad & Trat. & $\begin{array}{l}\text { Polifenoles totales } \\
\left(\mathrm{mg} \text { ác. gálico } \cdot \mathrm{g}^{-1}\right)\end{array}$ & $\begin{array}{l}\text { Flavonoides totales } \\
\left(\mathrm{mg} \text { catequina } \cdot \mathrm{g}^{-1}\right)\end{array}$ & $\begin{array}{c}\text { Capacidad } \\
\text { antioxidante por } \\
\text { método FRAP } \\
\left(\mu \mathrm{moltrolox} \cdot \mathrm{g}^{-1}\right)\end{array}$ & $\begin{array}{c}\text { Capacidad } \\
\text { antioxidante por } \\
\text { método ABTS } \\
\left(\mu \mathrm{moltrolox} \cdot \mathrm{g}^{-1}\right)\end{array}$ \\
\hline \multirow{4}{*}{$\begin{array}{l}\text { Pichincha- } \\
\text { Tumbaco }\end{array}$} & $\mathbf{P E}$ & $80,29 \pm 1,20$ & $75,24 \pm 2,94$ & $1144,76 \pm 32,29$ & $567,00 \pm 19,32$ \\
\hline & $\mathbf{T}_{1}$ & $44,03 \pm 0,85$ & $38,37 \pm 0,47$ & $949,01 \pm 3,17$ & $384,15 \pm 3,71$ \\
\hline & $\mathbf{T}_{2}$ & $37,55 \pm 0,65$ & $32,56 \pm 1,28$ & $728,14 \pm 9,89$ & $344,48 \pm 8,73$ \\
\hline & $\mathbf{T}_{3}$ & $93,56 \pm 1,51$ & $86,00 \pm 2,59$ & $1327,86 \pm 36,62$ & $800,16 \pm 39,01$ \\
\hline \multirow{4}{*}{$\begin{array}{l}\text { Imbabura- } \\
\text { Italquí }\end{array}$} & $\mathbf{P E}$ & $109,28 \pm 4,23$ & $95,66 \pm 1,86$ & $603,40 \pm 2,06$ & $497,32 \pm 0,14$ \\
\hline & $\mathbf{T}_{1}$ & $57,66 \pm 1,12$ & $48,88 \pm 6,31$ & $357,73 \pm 2,50$ & $378,54 \pm 0,39$ \\
\hline & $\mathbf{T}_{2}$ & $61,99 \pm 1,69$ & $55,21 \pm 0,74$ & $458,74 \pm 7,43$ & $381,76 \pm 3,20$ \\
\hline & $\mathbf{T}_{3}$ & $145,24 \pm 6,04$ & $131,71 \pm 2,03$ & $898,41 \pm 2,16$ & $897,14 \pm 11,02$ \\
\hline
\end{tabular}

PE: planta entera; $\mathbf{T}_{1}$ : raíz; $\mathbf{T}_{2}$ : tallo; $\mathbf{T}_{3}:$ hojas; $\mathrm{n}=6$

Con base en los resultados reportados en la Tabla 2, se establecieron los valores de la capacidad antioxidante expresada en concentración de trolox. El análisis de las muestras se realizó empleando dos métodos de cuantificación FRAP y ABTS ${ }^{+}$, y los resultados en la planta entera para el orégano de Tumbaco fue de $1.144,76$ y $567,00 \mu$ mol trolox $\mathrm{g}^{-1}$, respecti- vamente; para el orégano de Italquí los valores disminuyeron en 603,40 y 497,32 $\mu$ mol trolox $\mathrm{g}^{-1}$. Con base en estos resultados se observa que la planta de orégano que tiene una capacidad antioxidante mayor corresponde al ambiente de Tumbaco (Pichincha.), obteniéndose en las hojas el mayor contenido con 1.327,86 $\boldsymbol{\mu m o l}$ trolox $\mathrm{g}^{-1}$. 


\section{Discusión}

Antal et al. (2015) presentan un análisis del contenido de macrominerales en plantas de orégano de tres sitios de la parte sur de Rumania; de igual manera, Zagula et al. (2016) realizaron en análisis del contenido de minerales en diferentes tipos de hierbas secas, en ambos casos los investigadores obtuvieron para el orégano resultados similares a los encontrados en el presente trabajo.

En el mismo estudio, Antal et al. (2015) hacen referencia al contenido de microminerales en plantas de orégano; resultados que presentan valores similares a los obtenidos en esta investigación para cobre, manganeso y zinc; de igual manera, la investigación realizada en plantas medicinales y sus extractos por Kostić et al. (2011) reportan una similitud con los valores experimentales que se obtuvieron en este artículo. Casas Castro (2019) presenta un análisis de cómo los factores medioambientales, entre ellos la temperatura, luz, humedad relativa, el $\mathrm{pH}$ del suelo, controlan la absorción y translocación de los minerales por parte de la planta.

Debido a las condiciones ambientales diversas entre ambas localidades, el resultado del presente trabajo evidenció las diferencias significativas en contenido de macro y microminerales para morfotipo de orégano demostrándose la mayor cantidad en el cultivo de Italquí. Flores-Martínez et al. (2016) determinaron el contenido de polifenoles totales en las hojas del orégano, obteniendo un resultado cercano a los 101,0 mg ác. gálico $\cdot \mathrm{g}^{-1}$, que se asemeja con los valores obtenidos para las hojas del orégano de Tumbaco, superado por su contenido en las hojas de las muestras de Italquí. De igual manera, en el estudio de Shan et al. (2005) al evaluar la capacidad antioxidante obtuvieron 101,7 mg ác. gálico $\mathrm{g}^{-1}$ de polifenoles totales para las hojas del $O$. vulgare L. Mercado-Mercado et al. (2013) presentaron los resultados de los compuestos fenólicos y la capacidad antioxidante de algunas especias típicas consumidas en México; en el O. vulgare L., los resultados presentados para flavonoides totales en $\mathrm{mg}$ catequina $\cdot 100 \mathrm{~g}^{-1}$ exhiben similitud a los resultados obtenidos en la presente investigación. En el estudio de Za-
vala-Nigoa et al. (2010) de los extractos de las hojas de la especie de orégano (Lippia graveolens) determinaron el contenido de flavonoides totales con $163,75 \mathrm{mg}$ catequina $\mathrm{g}^{-1}$; sus resultados son superiores a los valores obtenidos en esta investigación.

Mercado-Mercado et al. (2013) en su investigación sobre capacidad antioxidante de las especias típicas de México empleando varios métodos de análisis (FRAP, ABTS $\cdot{ }^{+}$, ORAC y DPPH) obtuvieron resultados para una muestra de orégano, donde el valor de capacidad antioxidante obtenido por el método ABTS ${ }^{+}$fue de 1.007 $\mu \mathrm{mol}$ trolox $\cdot \mathrm{g}^{-1}$, resultando superior al obtenido experimentalmente en esta investigación. Vallverdú-Queralt et al. (2014) realizaron un estudio sobre el perfil fenólico de las hierbas y especias utilizadas, la capacidad antioxidante se determinó por el método $\mathrm{ABTS}^{+}{ }^{+}$y DPPH; para el caso puntual de la muestra de orégano, el valor de la capacidad antioxidante obtenido por ABTS ${ }^{+}$ fue $1.340 \mu \mathrm{mol}$ torolox $\cdot \mathrm{g}^{-1}$, valor superior a los obtenidos en el presente trabajo investigativo $\mathrm{y}$ que corresponde a las condiciones ambientales de Tumbaco. De acuerdo con Oracz \& Nebesny (2016), el contenido fenólico y, por ende, la capacidad antioxidante se ve frecuentemente afectada por factores geográficos, la época de cosecha y los días expuestos al acopio. La investigación realizada por Katsube et al., (2003) señala que la actividad antioxidante es también dependiente de la concentración del extracto obtenido.

\section{Conclusiones}

El estudio permitió comparar un morfotipo de orégano procedente de dos zonas del país. El análisis proximal indica que la presencia de macronutrientes como proteína, grasa y carbohidratos es superior en las hojas.

Los macrominerales abundan en el tallo y las hojas del orégano de ambas localidades. Los microminerales se encuentran en mayor cantidad en el morfotipo cultivado en Italquí, al igual que el contenido de polifenoles y flavonoides totales.

La capacidad antioxidante prevalece con mayor contenido en el morfotipo cultivado en las condiciones ambientales de Tumbaco. 


\section{Referencias}

Allauca, J., Tapia, C., Tacán, M., Monteros, A., \& Brito Grandes, B. (2013). Estudio de la biodiversidad de plantas medicinales en las provincias de Carchi, Imbabura y Pichincha. Quito: INIAP - Instituto Nacional de Investigaciones Agropecuarias, Departamento Nacional de Recursos Fitogenéticos.

Antal, D., Citu, C., Ardellan, F., Dehelean, C., Viaia, L., Soica, C., Viaia, V., Biris, M., \& Saas, I. (2015). Metallome of Origanum vulgare: the unknown side of a medicinal and aromatic plant used worldwide. FARMACIA, 63(4), 534-538.

Casas Castro, A. (2019). Factores ambientales que afectan la nutrición vegetal. Obtenido de; https:/www.himarcan.com/wp-content/ uploads/2019/11/Factores-Ambientalesque-Afectan-a-la-Nutricion-VegetalAntonio-Casas.pdf

Cross, E., Villenueve, F., \& Vicent, J. (1982). Recherche d'un indice de fermentation du cacao. Evolution des tanins et des phénols totaux de la feve. Cacao-Café, Thé Paris, XXVI(2), 109-114.

de la Torre, L., Navarrete, H., Muriel, P., Macía, M., \& Balslev, H. (2008). Enciclopedia de las plantas útiles del Ecuador, 1. ${ }^{\mathrm{a}}$ ed. Quito, Ecuador: Aarhus.

Flores-Martínez, H., León-Campos, C., Estarrón-Espinosa, M., \& Orozco-Ávila, I. (2016). Optimización del proceso de extracción de sustancias antioxidante a partir del orégano mexicano (L. graveolens HBK) utilizando la metodología de superficie de respuesta (MSR). Revista Mexicana de Ingeniería Química, 15(3), 773-785.

INIAP - Instituto Nacional de Investigaciones Agropecuarias. (2017). Laboratorio de servicio de análisis e investigación de alimentos del Instituto Nacional Autónomo de Investigaciones agropecuarias. Quito: INIAP.
Katsube, N., Iwashita, K., Tsushida, T., Yamaki, K., \& Kobori, M. (2003). Induction of apoptosis in cancer cells by Bilberry (Vaccinium myrtillus) and the anthocyanins. Journal of agricultural and food chemistry, 51(1), 6875. https://doi.org/10.1021/jf025781x

Koksal, O., Ozer, O., \& Muzaden, M. (2010). Analysis of effective factors on information sources at Turkish Oregano farms. African Journal of Agriculture Research, 5(2), 142149. https://doi.org/10.5897/AJAR09.598

Kostić, D., Mitić, A., Zarubica, A., Mitić, M., Veličković, J., \& Randjelichić, S. (2011). Content of trace metal in medicinal plants and their extracts. Hemijska Industrija,, 65(2), 165-170. https://doi.org/10.2298/ HEMIND101005075K

Mercado-Mercado, G., Carrillo, L., Wall-Medrano, A., López, J., \& Álvarez-Parrilla, E. (2013). Compuestos polifenólicos y capacidad antioxidante de especies típicas consumidas en México. Nutrición Hospitalaria, 28(1), 36-46. http://dx.doi.org/10.3305/ nh.2013.28.1.6298

Oracz, J., \& Nebesny, E. (2016). Antioxidant properties of cocoa beans (Teobroma cacao L.): Influence of cultivar and roasting conditions. International Journal of Food Properties, 19(6), 1242-1258. https://doi.or g/10.1080/10942912.2015.1071840

Re, R., Proteggente, N., Pannala, A. Y., \& Rice-Evans. (1998). Antioxidant activity applying an impoved ABTS + radical Cation decolorization assay. Free Radical Biology and Medicine, 26(9-10), 1231-1237. https:// doi.org/10.1016/S0891-5849(98)00315-3

Shan, B., Cai, Y. Z., Sun, M., \& Corke, H. (2005). Antioxidant capacity of 26 spice extracts and characterization of their phenolic constituents. Journal of agricultural and food chemistry, 53(20), 7749-7759. https://doi.org/10.1021/jf051513y 
Vallverdú-Queralt, A., Raquiero, J., Martínez-Huelamo, M., Rinaldi, J., Leal, L., \& Lamuela-Raventos, R. (2014). A comprehensive study on the phenolic profile of widely used culinary herbs and spices: rosemary, thyme, oregano, cinnamon, cumin and bay. Food Chemistry, 154(1), 299-307. https://doi.org/10.1016/j.foodchem.2013.12.106

Yen, G.-C., \& Chen, H.-Y. (1995). Antioxidant activity of varius tea extracts in relation to their antimutagenicity. Journal Agriculture Food Chemistry, 43(1), 27-32. https://doi. org/10.1021/jf00049a007

Zagula, G., Fabisiak, A., Bajar, M., Czernicka,M., Saletnik, B., \& Puchalski,C. (2016). Mineral components analysis of selected dryed hebrs. ECONTECHMOD: An International Quar- terly Journal on Economics of Technology and Modelling Processes, 5(2), 127-132.

Zavala-Nigoa, J., Loarca-Piña, G., \& García-García, T. (2010). Evaluación del contenido fenólico, capacidad antioxidante y actividad citotóxica sobre células caco-2 del extracto acuoso de orégano (Lippia graveolens KUNT). 2. ${ }^{\circ}$ Congreso Nacional de Química Médica. Querétaro.

Zhishen, J., Mengcheng, T., \& Jianming, W. (1999). The determination of flavonoid contents in mulberry and their scavenging effects on superoxide radical. Food Chemisry, 64(4), 555-559. https://doi.org/10.1016/ S0308-8146(98)00102-2 